\title{
artigo
}

\section{Análise do percentual de desperdício do leite humano e de fórmulas infantis em serviço de neonatologia}

\author{
Analysis of the percentage of waste from human milk and child formulas in neonatology service \\ Análisis del porcentaje de residuos de leche humana y fórmulas infantiles en el servicio de neonatología
}

\begin{abstract}
RESUMO
Objetivo: avaliar o desperdício de leite materno e fórmula infantil em serviço de neonatologia. Método: estudo transversal, realizado no período de junho a dezembro de 2019 em uma maternidade escola de Natal-RN. A análise descritiva das variáveis contínuas foi realizada pela mediana e percentis. Para as variáveis categóricas a análise foi realizada por meio de frequências absolutas e relativas. Foi aplicado o teste de Mann-Whitney e o teste Qui-quadrado. Resultado: houve maior desperdício de fórmula infantil na Unidade de Terapia Intensiva Neonatal $(p<0,01)$ e a justificativa mais comum para o não aproveitamento da fórmula foi a necessidade de procedimento nos neonatos. Conclusão: considerando a importância do leite materno e fórmula infantil para os recém-nascidos hospitalizados nas unidades estudadas, alertamos que se faz necessário um maior controle das distribuições dessas dietas em função do impacto na saúde infantil e nos custos hospitalares.
\end{abstract}

DESCRITORES: Leite materno; Fórmulas infantis; Desperdício de alimentos.

\section{ABSTRACT}

Objective: to evaluate the waste of breast milk and infant formula in a neonatology service. Method: cross-sectional study, carried out from June to December 2019 in a maternity school in Natal-RN. The descriptive analysis of continuous variables was performed by median and percentiles. For categorical variables, the analysis was performed using absolute and relative frequencies. The Mann-Whitney test and the Chi-square test were applied. Result: there was a greater waste of infant formula in the Neonatal Intensive Care Unit $(p<0.01$ ) and the most common justification for not using the formula was the need for a procedure in neonates. Conclusion: considering the importance of breast milk and infant formula for newborns hospitalized in the units studied, we warn that greater control of the distributions of these diets is necessary due to the impact on child health and hospital costs

DESCRIPTORS: Breast milk; Infant formulas; Food wast.

\section{RESUMEN}

Objetivo: Evaluar el desperdicio de leche materna y fórmula infantil. Método: estudio transversal, realizado de junio a diciembre de 2019 en una escuela de maternidad en Natal-RN. El análisis descriptivo de las variables continuas se realizó por mediana y percentiles. Para las variables categóricas, el análisis se realizó mediante frecuencias absolutas y relativas. Se aplicó la prueba de Mann-Whitney y la prueba de Chi-cuadrado. Resultado: Hubo un mayor desperdicio de fórmula infantil en la Unidad de Cuidados Intensivos Neonatales ( $\mathrm{c}$ 0.01) y la justificación más común para no usar la fórmula fue la necesidad de un procedimiento en el recién nacido. Conclusión: Considerando la importancia de la leche materna y la fórmula infantil para los recién nacidos hospitalizados en las unidades estudiadas, advertimos que es necesario un mayor control de las distribuciones de estas dietas por el impacto en la salud infantil y los costos hospitalarios.

DESCRIPTORES: Leche materna; Fórmulas para lactantes; Desechos alimentarios.

RECEBIDO EM: 24/11/2020 APROVADO EM: 08/12/2020

\section{Letícia Costa Ferreirra}

Especialista, nutricionista, residente do Programa de Residência Multiprofissional em Intensivismo Neonatal da Maternidade Escola Januário Cicco - MEJC/UFRN, Natal/RN.

ORCID: 0000-0002-7786-7214

\section{Ana Verônica Dantas de Carvalho}

Mestre, nutricionista da Maternidade Escola Januário Cicco - MEJC/UFRN, Natal/RN.

ORCID: 0000-0002-8840-6368 


\section{Juliana Dantas de Araújo Santos Camargo}

Mestre, estatística da Maternidade Escola Januário Cicco - MEJC/UFRN, Natal/RN.

ORCID: 0000-0001-8692-5706

\section{Anna Christina do Nascimento Granjeiro Barreto}

Doutora, médica intensivista da Maternidade Escola Januário Cicco - MEJEC/UFRN, Natal/RN.

ORCID: 0000-0001-7319-5011

\section{Edienne Rosângela Sarmento Diniz}

Doutoranda do Programa de Pós Graduação em Saúde Coletiva da UFRN, enfermeira intensivista neonatal da Maternidade Escola Januário Cicco - MEJC/UFRN, Natal/RN.

ORCID: 0000-0001-5546-023X

\section{Maria do Livramento Silva Bitencourt}

Mestre, enfermeira do Hospital Universitário Lauro Wanderley, UFPB/EBSERH. ORCID: 0000-0002-8807-2740

\section{INTRODUÇÃO}

0 suporte nutricional é indispensável ao recém-nascido hospitalizado, sendo fundamental para melhoria das alterações metabólicas nas primeiras 24 a 48 horas de internação ${ }^{1}$. Neste contexto, o leite materno (LM) é considerado um alimento completo o qual traz benefícios ao sistema digestivo, sistema nervoso, imunológico, mantém o crescimento e desenvolvimento normal da criança e impede a adesão e colonização de patógenos bacterianos respiratórios, entre outros ${ }^{2}$.

Apesar de todas as evidências científicas desses benefícios, na realidade brasileira, principalmente nas regiões Norte e Nordeste, os índices de amamentação exclusiva ainda estão abaixo do recomendado pela Organização Mundial de Saúde (OMS) e pelo Ministério da Saúde, conforme divulgado pelo Estudo Nacional de Alimentação e Nutrição Infantil (Enani), apenas $47 \%$ das crianças receberam amamentação exclusiva durante os seis primeiros meses e $60 \%$ até os quatro meses de vida ${ }^{3}$.

Almejando melhorar essa realidade, desde 1981 que foram criados os Bancos de Leite Humano (BLH), responsáveis pela coleta, processamento, controle de qualidade e distribuição a uma população vulnerável, regulamentados pela RDC 171 de 4 de setembro de 2006. No entanto, o volume de LM doado disponível nos bancos ainda é insuficiente para suprir toda a demanda existente. Um estudo realizado em BLH de um hospital público do Paraná verificou que dos 402.7 litros de leite doado, 61,5L foram desprezados (15\%), tendo como principal motivo a presença de sujidade. Os autores alertaram para importância de investimento na capacitação dos profissionais a fim de otimizar o aproveitamento do LM contribuindo para redução de gastos com fórmulas artificiais e com a mortalidade infantil ${ }^{4}$.

As vantagens do $L M$ sobre as fórmulas infantis (FI) são inegáveis, no entanto, existem situações em que o uso dessas fórmulas são necessárias: no contexto de inviabilidade de utilização do leite materno, por contraindicação clínica da lactação, ou em situações de risco, como por exemplo, mães portadoras do Vírus da Imunodeficiência Humana (HIV) ${ }^{5}$ ou ainda para complementação do aporte calórico do RN4. Na instituição estudada, a qual faz parte da Iniciativa Hospital Amigo da criança (IHAC), é priorizada a oferta do leite materno cru ou pasteurizado aos recém-nascidos (RNs) da Unidade de Terapia Intensiva Neonatal (UTIN) e Unidade de Cuidados Intermediários Neonatais Canguru (UCINca), visto que os RNs hospitalizados nessas unidades se encontram impossibilitados de mamar ao seio materno e dependentes do aporte nutricional do lactário.

Neste interim, cientes da importância do leite materno e das fórmulas infantis para os RNs hospitalizados, bem como do custo institucional da dietoterapia neonatal, questiona-se: existem desperdícios de dietas na unidade pesquisada? Quais os fatores geradores desses desperdícios?
Assim, este estudo teve como objetivo avaliar a existência de desperdícios e quais os fatores geradores destes, em uma Maternidade Escola do Município de Natal/RN.

\section{MÉTODO}

Trata-se de um estudo transversal realizado no período de junho a dezembro de 2019. A pesquisa foi realizada no lactário de uma maternidade escola de Natal/RN. Os dados utilizados na coleta foram secundários, oriundos dos livros de registros de dispensação de dietas do lactário da instituição pesquisada. Foram extraídas as seguintes variáveis de interesse: quantidade de dieta total distribuída $(\mathrm{ml})$, quantidade de dieta total desperdiçada $(\mathrm{ml})$, setor de distribuição e motivos do descarte de dietas (ocorrência de procedimento, amamentação, intercorrência e outros).

Após a coleta dos dados, estes foram armazenados em uma planilha eletrônica no software SPSS for Windows, versão 22.0. Utilizou-se o teste de normalidade de Kolmogorov-Smirnov para verificar a aderência das variáveis contínuas à distribuição normal. Para as variáveis que não apresentaram distribuição normal, utilizou-se a mediana, os percentis 25 e 75 , além do teste não paramétrico de Mann-Whitney. A análise das variáveis categóricas foi realizada por meio de frequências absolutas e relativas. $\mathrm{O}$ teste Qui-quadrado foi utilizado para analisar a associação entre as variáveis de natureza categórica. Em todas as análises foi adotado o nível de 5\% de significância. 


\section{artigo}

Ferreira, L.X.; Carvalho, A.V.D.; Carmargo, J.D.A.S.; Barreto, A.C.N.G.; Diniz, E.R.S.; Bitencourt, M.L.S.;

Análise do percentual de desperdício do leite humano e de fórmulas infantis em serviço de neonatologia

$\mathrm{O}$ estudo atendeu as recomendações da Resolução No466/2012 que trata da pesquisa que envolve seres humanos, sendo apreciada pelo Comitê de Ética em Pesquisa do Hospital Onofre Lopes (HUOL) e aprovada conforme parecer CAAE nº 5634019.2.0000.5292.

\section{RESULTADOS}

Foi analisada a comparação do percentual de desperdício entre os setores UTIN e UCINca das 424 ofertas de leite e de fórmulas ocorridas durante 212 dias, sendo observado maior nível de desperdício na UTIN para fórmula infantil $(\mathrm{p}<0,01)$, conforme demonstrado na tabela 1.

Quando investigado os motivos geradores de desperdício de leite materno e fórmula infantil nos setores, observou-se os seguintes resultados: na UTIN o leite materno e a fórmula infantil foram desperdiçados principalmente pela ocorrência de procedimento no neonato em 33 e $39 \%$ dos casos, respectivamente; já na UCINca, $42 \%$ da fórmula infantil deixou de ser aproveitada também pela necessidade de procedimento no RN e $40 \%$ do leite materno por outros motivos, conforme evidenciado na figura 1 .

\section{DISCUSSÃO}

Neste estudo, observou-se uma maior frequência de desperdícios de dietas distribuídas na UTIN sob a justificativa de realização de procedimentos nos RNs. A UTIN é um setor caracterizado por possuir dinâmicas próprias e complexas, onde o cuidado prestado exige competências especializadas e processos de trabalho estabelecidos por protocolos clínicos e assistenciais6. Essa dinâmica intensa, com elevado número de procedimentos/dia realizados associado ao subdimensionamento de enfermagem pode ter contribuído para o maior

\section{Tabela 1- Análise comparativa do desperdício de dietas na UTIN e UCINca, no período de junho a dezembro de 2019.} Natal-RN, 2019.

Variáveis

Leite materno distribuído (ml)

Leite materno desperdiçado (ml)

Percentual de leite materno desperdiçado

Fórmula infantil distribuída (ml)

Fórmula infantil desperdiçada (ml)

Percentual de Fórmula infantil desperdiçada
Setor de distribuição

$$
\text { UTIN UCINca }
$$

$1.980(1.736-2.232)$

$944(612-1.278)$

$32(18-54)$

$30(20-37)$

$1,18 \%$

$0,31 \%$

2.301 (1.806 - 2.968)

1.594 (1.089-

1.926)

$$
54(45-75)
$$

$33(19-41)$

$0,87 \%$

$0,14 \%$

P valor $^{\mathrm{a}}$

Total

asignificância da diferença entre os grupos pelo teste Mann-Whitney. Percentual do leite materno desperdiçado = quantidade total do leite materno (ml) desperdiçado/ quantidade total do leite materno (ml) distribuído. Percentual da fórmula desperdiçada = quantidade total da fórmula infantil (ml) desperdiçada/ quantidade total da fórmula infantil (ml) distribuída. Os dados contínuos estão expressos em mediana e percentil 25 (P25) e percentil 75 (P75): Mediana (P25-P75).

Valores em negrito indicam significância em $p<0,05$.

Abreviações: UTIN: Unidade de Terapia Intensiva Neonatal; UCINca: Unidade de Cuidados Intermediários Neonatais Canguru; ml: mililitros.

Fonte: livros de registros de dispensação de dietas do lactário da instituição pesquisada.

\section{Figura 1 - Distribuição percentual dos motivos de desperdício do leite materno e fórmula infantil, por setor. Natal-RN, 2019.}

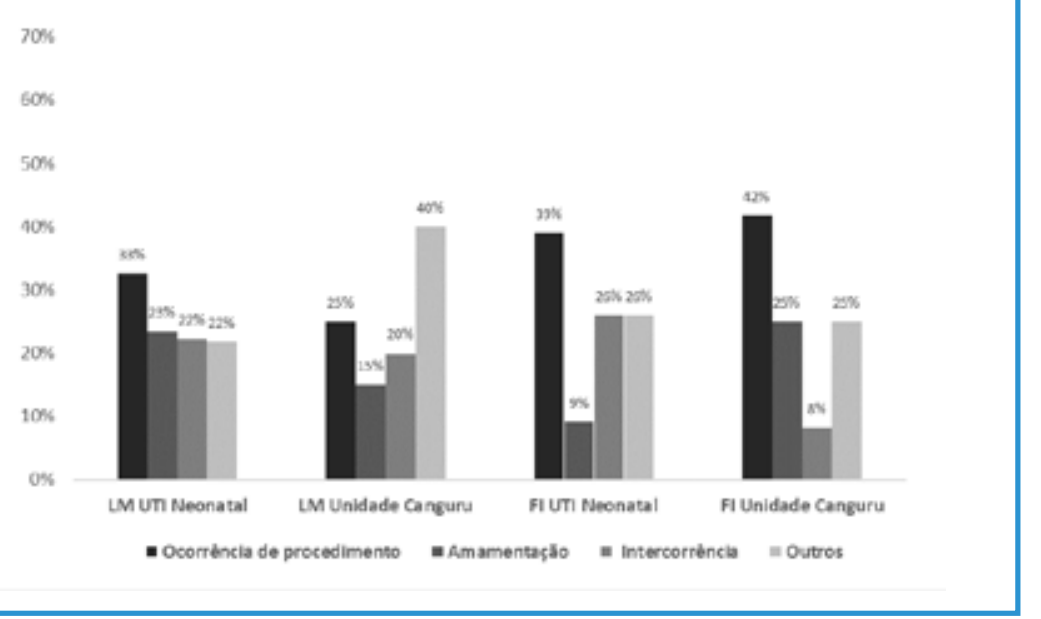

LM- leite materno; FI- fórmula infantil

Fonte: livros de registros de dispensação de dietas do lactário da instituição pesquisada. desperdício de dietas na UTIN. Tal achado corrobora com outros estudos os quais apontam que o quantitativo subestimado de profissionais de enfermagem em UTIs, relaciona-se especialmente com a fragilização de atividades de gestão do cuidado, sobrecarga de trabalho e, consequentemente, possíveis repercussões negativas na qualidade e segurança da assistência ${ }^{7}$, o que inclui a dificuldade da comunicação com o lactário para avisar sobre novas prescrições o que acaba gerando o desperdício das dietas.

Sabe-se que apesar do serviço possuir um banco de leite humano (BLH) que abastece o lactário da instituição, o quantitativo de LHP dispensado é insuficiente para atender as demandas da instituição. Neste cenário, a cada ml de leite humano desperdiçado uma vida pode ser prejudicada, uma vez que o aleitamento materno está relacionado com 
a melhora clínica do $\mathrm{RN}$ e com a redução do tempo de hospitalização ${ }^{8,9}$.

$\mathrm{O}$ uso de fórmulas infantis é indicado quando o uso do leite materno é inviável, no entanto, devemos nos atentar ao fato de que o custo das fórmulas infantis é elevado, principalmente quando se trata de fórmulas especializadas para $\mathrm{RNs}$ prematuros e alérgicos. Segundo Oldenburg4 a existência de desperdício implica no aumento de custos, e sabe-se que quando se trata de saúde pública, um dos principais pilares para mudança de qualquer prática é a necessidade de redução de gasto financeiro. A redução de gastos para os cofres públicos é relevante, particularmente em momentos em que os recursos são escassos, sendo que no Brasil, atualmente, menos de $4 \%$ do PIB é investido na saúde pública ${ }^{10,11}$.

Os motivos de desperdícios de dietas na UTIN e na UCINca estão associados a ocorrência de procedimentos, amamentação e intercorrências com os RNs. Tais achados revelam falhas no fluxo de distribuição de dietas. Solfa ${ }^{12}$ e Siqueira et al., ${ }^{13}$ encontraram em seus estudos falhas no processo relacionadas a não administração dos volumes de dietas enterais prescritas, em decorrência de suspensão, não cumprimento das ações prescritas referentes à terapia enteral no tempo preestabelecido, mudanças constantes nos horários das dietas, interrupção da dieta para a prática de outros procedimentos e o dimensionamento inadequado de pessoal.

Observa-se que os achados dessa pesquisa, bem como dos estudos citados mostram a necessidade de melhoria no processo de trabalho interdisciplinar nas instituições de saúde. Ademais, é nítida a dificuldade na articulação entre o setor do lactário com os profissionais da UTIN e da UCINca, fato que revela a necessidade de um fluxo de dietas na instituição pesquisada, que garanta a sustentabilidade do serviço sem perdas significantes. Acredita-se que fomentar o diálogo interdisciplinar entre os profissionais possa colaborar para a redução do desperdício.

\section{CONCLUSÃO}

Os desperdícios de leite materno e de fórmula infantil ocorre em ambos os setores estudados, sendo de maior significância no setor de Unidade de Terapia Intensiva Neonatal, tendo como principal motivo a realização de procedimento no neonato. Considerando a importância de suporte nutricional adequado aos recém nascidos, bem como a necessidade de otimização dos recursos financeiros institucional, alertamos que se faz necessário um melhor controle das distribuições de dietas através do estabelecimento de um fluxo institucional que resulte na redução do desperdício evidenciado.

\section{REFERÊNCIAS}

1. McClave S, Taylor B, Martindale RG, Warren MM, Johnson DR, Braunschweig C, McCarthy MS, Davanos E, Rice TW, Cresci GA, Gervasio JM, Sacks GS, Roberts PR, Compher C, Society of Critical Care Medicine; American Society for Parenteral and Enteral Nutrition. Guidelines for the Provision and Assessment of Nutrition Support Therapy in the Adult Critically III Patient: Society of Critical Care Medicine (SCCM) and American Society for Parenteral and Enteral Nutrition (A.S.P.E.N.). JPEN J Parenter Enteral Nutr. 2016; 40 (2):159-211.

2. Gonçalvez RM, Melo CS. Aleitamento materno versus aleitamento artificial. Especial. Goiânia: Pontifícia Universidade Católica de Goiás (PUC); 2014.

3.OPAS. Brasil. Disponivel em: https:/www.paho.org/bra/index. php?option=com_content\&view=article\&id=6242:brasil-lanca-campanha-de-amamentacao-durante-semana-mundial-do-aleitamento-materno\&ltemid=839. [Acesso em 02 de dez 2020]

4. Oldenburg L, Strasburg VJ, Reinheimer SM, Santos VR, Silva SM. Frequência de distribuição de leite materno e fatores associados em pacientes de hospital público. Revista Saúde (Sta. Maria) [periódico on line]. 2018 [acesso em 24 de set 2020]. 44 (2).

5. Silva AML, Monteiro GRSS, Tavares ANS, Pedrosa ZVRS. A introdução alimentar precoce e o risco de alergias: Revisão da literatura. Enferm Global -Esp- [periódico on line]. 2019 [acesso em 24 de set 2020]. 54: 485-498. Disponivel em: http:/scielo.isciii.es/pdf/eg/v18n54/ pt_1695-6141-eg-18-54-470.pdf.

6. Donoso MTV, Souza MAF, Mattos SS, Campos DMP, Silqueira SMF, Sharry S. A enfermagem nas unidades de terapia intensiva: o aparato tecnológico versus a humanização da assistência. RECOM [periódico on line]. 2017 [acesso em 24 de set 2020]. 7:e1883. Disponivel em:

http://seer.ufsj.edu.br/index.php/recom/article/view/1883/1794.
7. Carvalho EMP, Moraes KG. Consequências do subdimensionamento de pessoal na saúde dos trabalhadores da enfermagem. R. G\&S [periódico on line]. 2013 [acesso em 24 de set 2020]. 4 (4): 1556-1570. Disponivel em: https:/core.ac.uk/download/pdf/231162375.pdf.

8. Medeiros AMC, Oliveira ARM, Fernandes AM, Guardachoni GAS, Aquino JPSP, Rubinick ML, Zveibil NM, Gabriel TCF. Caracterização da técnica de transição da alimentação por sonda enteral para seio materno em recém-nascidos prematuros. J Soc Bras Fonoaudiol [periódico on line]. 2010 [acesso em 24 de set 2020]. 23 (1): 57-65.

9. Cristofalo EA, Schanler RJ, Blanco $C L$, Sullivan $S$, Trawoeger $R$, Kiechl-Kohlendorfer U, Dudell G, Rechtman DJ, Lee ML, Lucas A, Abrams S. Randomized trial of exclusive human milk versus preterm formula diets in extremely premature infants. J Pediatr [periódico on line]. 2013 [acesso em 24 de set 2020]. 163 (6):1592-95.

10. Johnson TJ, Patel AL, Bigger HR, Engstrom JL, Meier PP. Economic benefits and costs of human milk feedings: a strategy to reduce the risk of prematurity-related morbidities in very-low-birth-weight infants. Adv. Nutr. [periódico on line]. 2014 [acesso em 24 de set 2020]. 5 (2): 207-212.

11. Bartick MC, Stuebe AM, Schwarz EB, Luongo C, Reinhold AG, Foster EM. Cost analysis of maternal disease associated with suboptimal breastfeeding. Obstet Gynecol. 2017; 122 (1):111-9.

12. Solfa FV. Desperdício de dieta enteral em UTI: análise de modo de falhas e efeitos de danos ao paciente grave [dissertação]. Botucatu: Universidade Estadual Paulista "Júlio de Mesquita Filho"; 2014.

13. Siqueira CL, Siqueira FF, Lopes GC, Gonçalves MC, Sarantopoulos A. Enteral diet therapy: use of the Lean Healthcare philosophy in process improvement. Rev Bras Enferm [periódico on line]. 2019 [acesso em 24 de set 2020]. 72 (Suppl 1): 235-42. 